\title{
Impact of Nutrition and Depression on the Cognitive Functioning of the Elderly in Taiwan
}

Cheng-Fen Chang ${ }^{1 *}$ and Tien-Ho Kuo ${ }^{2}$

${ }^{1}$ Ching Kuo Institute of Management and Health, Taiwan

${ }^{2}$ Tungnan University, Taiwan

*Corresponding author: Cheng-Fen Chang, Ching Kuo Institute of Management and Health, Taiwan, E-mail: cfchang222@gmail.com

Received date: August 26, 2018; Accepted date: September 19, 2018; Published date: September 21, 2018

Copyright: $@ 2018$ Chang CF, et al. This is an open-access article distributed under the terms of the Creative Commons Attribution License, which permits unrestricted use, distribution, and reproduction in any medium, provided the original author and source are credited.

\begin{abstract}
Background: According to the Alzheimer's Disease International (ADI), the regional distribution of new dementia cases worldwide in 2015 shows an increased proportion of new cases arising in Asia, the Americas and Africa. Moreover, an average of one new case of dementia every three seconds is estimated, with prevalence increasing with age. Nutritional disorder is a risk factor in reversible dementia, and literature also points out that the prevalence of depression in dementia patients is $22 \%$, rendering depression a likely risk factor for future dementia. Therefore, this study mainly aimed to explore the relationship between nutrition and depression and cognitive function, and hopes that the findings can be used as a reference for providing medical care to the elderly.
\end{abstract}

Methods: This study used a cross-sectional survey to study the elderly in northern Taiwan. A total of 245 subjects were selected using convenience sampling, and a questionnaire survey was administered. The research tool was a structured questionnaire constructed by the researchers, and the results were analyzed using the SPSS 20.0 statistical software.

Results: A total of 245 elderly people over the age of 65 completed the questionnaire survey. According to the Pfeiffer's Short Portable Mental State Questionnaire (SPMSQ), 233 respondents (95.1\%) showed normal cognitive functioning, and 12 respondents $(4.9 \%)$ showed mild to serious cognitive impairment. The mean age of the subjects was 72.82 years old, with a standard deviation of \pm 7.80 . $92(37.6 \%)$ of the subjects self-reported normal health condition; average total cognitive score was $9.13( \pm 1.37)$; the average number of chronic diseases was $0.98( \pm$ $1.04)$; the average nutritional score was 25.23 ( \pm 3.71$)$; and the average total depression score was $1.56( \pm 0.65)$. Most of the respondents were women, had university education or above, were non-smokers, "thought about regularly exercising 3 times a week for 30 minutes each" and "agreed to regularly do trans theoretical exercises". However, these variables were not significantly correlated to cognitive functioning. The relationship between age and cognitive functioning was also statistically insignificant. The number of chronic diseases showed significant negative correlation, indicating that subjects with more chronic diseases showed lower cognitive scores. Total cognitive score showed significant positive correlation to nutritional status, indicating that the better the nutritional condition of the elderly, the better their cognitive functioning. Total cognitive score negatively correlated to total depression score, indicating that those with higher depression score had poorer cognitive functioning.

Conclusion: This study showed that nutritional status, depression and chronic diseases were important variables that affect cognitive functioning. In order to improve the cognitive functioning of the elderly, the prevention of these key risk factors (nutritional status, depression, chronic diseases) can be strengthened to reduce rapid deterioration in cognitive functioning.

Keywords: Elderly; Cognitive functioning; Nutrition; Depression

\section{Introduction}

Cognitive functioning is defined as the manifestation of the mental state of an individual through perception, memory, problem solving and expression abilities. Brain function, physical and mental health, and adaptability to changes in the external environment or psychosocial stress can result in cognitive functioning changes in an individual $[1,2]$.

Nutritional disorders are a risk factor in reversible dementia, and literature shows that the prevalence of depression in dementia patients is $22 \%$, thus rendering depression a likely risk factor for future dementia $[3,4]$.
According to the Alzheimer's Disease International (ADI), the regional distribution of new dementia cases worldwide in 2015 shows an increased proportion of new cases arising in Asia, the Americas and Africa [5,6]. Moreover, an average of one new case of dementia every three seconds is estimated, with prevalence increasing with age. In Taiwan, the Ministry of Health and Welfare reported that among those 65 years old or above, the prevalence rate for dementia is about $8.04 \%$, and for mild cognitive impairment, about $18.76 \%$ [5-8]. According to CEPD mentioned in 2012, an average increase of 5 years each increases the prevalence rate by one times [9].

Based on the national population growth reported in the Republic of China Population Projection (2014-2061) by the National Development Council (NDC) in August 2014, and the 5-year dementia 
prevalence, the Taiwan Alzheimer's Disease Association estimated that in 2031, the number of people with dementia would have exceeded 470,000 , with more than 2 cases per 100 people. In 2041, the number of people with dementia is estimated to exceed 680,000 , with more than 3 cases per 100 people; in 2051, exceed 860,000, with more than 4 cases per 100 people; in 2061, exceed 930,000, with more than 5 cases per 100 people. In the next 47 years, dementia in Taiwan will have grown at an average rate of 40 new cases per day [10].

In an aging society, reducing dementia in the elderly is an urgent issue. Therefore, delaying decline in cognitive functioning and improving healthy body functions among the elderly are critical. As such, determining the impact of nutrition and depression on the cognitive functioning of the elderly is extremely crucial. Therefore, this study aimed to explore the impact of nutrition and depression on the cognitive functioning among the elderly in northern Taiwan to better understand the status of cognitive functioning among the elderly in that region. At the same time, this study aimed to examine the sociodemographic, nutritional and depression variables in cognitive functioning. It is hoped that based on the findings, an appropriate empirical study for cognitive function intervention can be developed for the elderly in the community, and serve as a reference for elderly cognitive function policy planning and implementation by the health administration. Therefore, the research goals are:

(1) Determine the current socio-demographic, nutritional, exercise, and depression variables and cognitive functioning statuses of the subjects.

(2) Examine the relationship between socio-demographic, nutritional, exercise, depression variables and the cognitive functioning of the subjects.

\section{Material and Methods}

\section{Research framework}

Based on the research goals and domestic and international literature, the research framework (Figure 1) mainly explored the relationship between socio-demographic, nutritional and depression variables, and cognition.

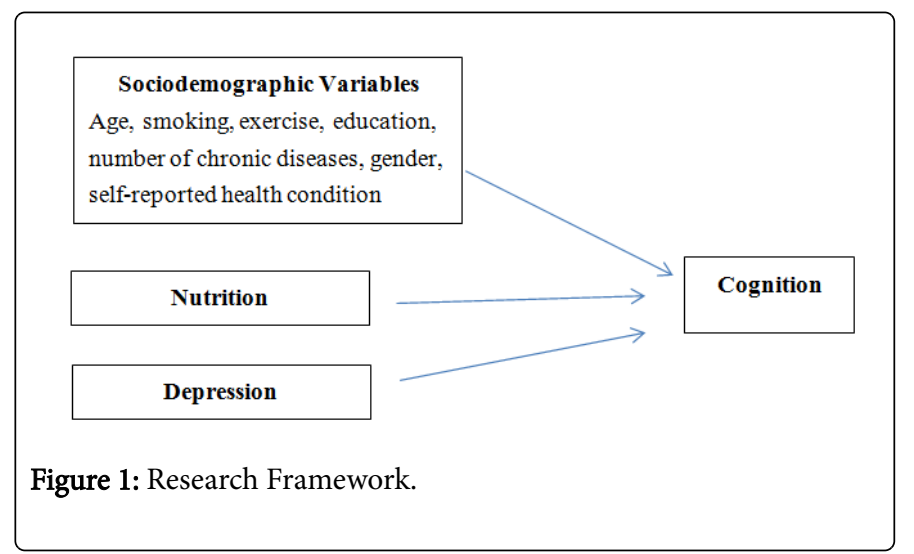

\section{Research subjects and data collection}

Using convenience sampling, elderly people aged 65 years old or above living in northern Taiwan was selected as study subjects. Interviewers trained in the questionnaire survey visited the subjects in their homes to explain the research goals and the scope of data use being limited to the research only. Then with consent, the interview was conducted anonymously. In case of discomfort during the research process, the subjects were free to withdraw at any time. At the end, no subject was deleted from the study sample, and a total of 245 valid samples were obtained.

\section{Research tools}

This study is a cross-sectional survey. The preliminary draft of the structured questionnaire was constructed according to the research framework and relevant literature, and the official version was finalized after expert validity review, pre-test and revision. The questionnaire included socio-demographic (age, smoking, exercise, education, number of chronic diseases, gender and self-reported health condition) factors, depression, nutrition, and cognitive status. The reliability test of each questionnaire subscale showed a Cronbach's a coefficient of .81 to .88 , indicating that each questionnaire subscale had good internal consistency.

(1) Basic information: Included age, smoking, exercise, education, number of chronic diseases, gender and self-reported health condition.

(2) The geriatric depression scale: The Geriatric depression scaleshort form (CT-GDS-SF) is a 15-item self-report scale where each respondent is only required to answer "Yes" or "No" to assess for symptoms of depression in the past week [10]. A "Yes" response to positively worded items is given one point while a "No" response is given 0 points. On the other hand, a "No" response to negatively worded items is given one point while a "Yes" response is given 0 points. The total score is 15 points, and the critical score is 5 points, where a score greater than 5 points indicates symptoms of depression and the higher the score, the more severe the depression. The scale has a Cronbach's $\alpha$ of 0.83 (which is greater than 0.7 ), indicating that the questionnaire result is highly reliable.

(3) Mini nutrition assessment scale (MNA): This study used the Mini Nutritional Assessment Taiwan Version-2 (MNA-T2-SF) as a measurement tool to assess the nutritional status of the subjects. The scale contains 18 questions [11]. The total score is 30 points, and a score $\geqq 24$ points indicates good nutrition; $17-23.5$ points, nutritional risk; and $\leqq 16.5$, malnutrition.

(4) Short portable mental state Questionnaire (SPMSQ): This study used the Short Portable Mental State Questionnaire (SPMSQ) (Pfeiffer, 1975) as a tool to measure the degree of cognitive functioning in the subjects. The questionnaire content includes consciousness, memory, orientation, attention, thinking and general knowledge. The questionnaire comprises a total of 10 items, with 1 point per correct answer and 0 points per wrong answer. The total score is 10 points. A score of 7-10 points indicates normal cognitive functioning while $\leqq 6$ points indicates impaired cognitive functioning..

\section{Data analysis}

The study data were collected through individual interviews, then encoded, decoded and documented into a database for analysis using SPSS for Window 20.0 statistical software. Statistical methods included frequency distribution tables, percentages, mean, standard deviation, $\mathrm{t}$ test, One-way ANOVA, and Pearson product-moment correlation analysis. 


\section{Results and Discussion}

\section{Distribution of social demographic variables}

Of the 245 study subjects, 76 (31\%) were male and 169 (69\%) were female. The mean and standard deviation for age were $72.82( \pm 7.80)$. In terms of education, 7 subjects $(2.9 \%)$ were illiterate; 57 subjects (23.3\%), elementary school education; 36 subjects (14.7\%), middle school education; 64 (26.1\%), high school education; and 81 (33.1\%), university education or above. In terms of self-reported health condition, 11 subjects (4.5\%) felt very unhealthy; 43 (17.6\%), unhealthy; 92 (37.6\%), average health; 57 (23.3\%), healthy; and 42 (17.1\%), very healthy. For smoking, 185 subjects (75.5\%) never smoked; 13 subjects (5.3\%) smoked occasionally; 27 (11\%) smoked daily; and $20(8.2 \%)$ had stopped smoking. The mean and standard deviation for the number of chronic diseases were $0.98( \pm 1.04)$. In terms of exercising 3 times weekly for 30 minutes each, 35 subjects (14\%) persisted for 6 months, 29 (12\%) persisted for less than 6 months, 35 (14\%) were getting ready to exercise, 78 (32\%) were thinking about it, and 68 (28\%) had never thought about it (Figure 2); 35 subjects (14\%) implemented regular trans theoretical exercises for more than 6 months, 29 (12\%) exercised for less than 6 months, 107 (44\%) agreed to exercise regularly, and $74(30 \%)$ did not agree to exercise regularly (Figure 3 ).

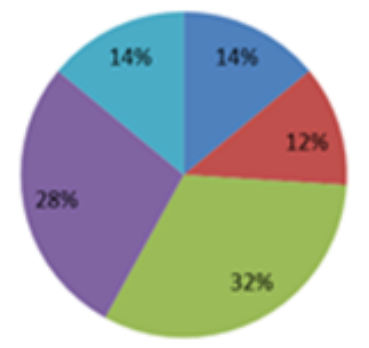

more than 6 months

Iless than 6 months

E thought

anever thought

in prepared

Figure 2: The weekly movement 3 times for 30 minutes each.

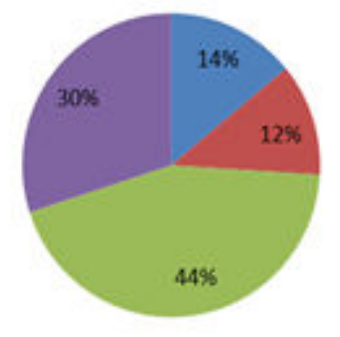

more than 6 months

= less than 6 months

wagree with regular moment

ndisagree with regular moment

Figure 3: The regular movement stage of trans theoretical model.

\section{Nutritional status}

The average total nutritional score was 25.23 ( \pm 3.71$)$. In the last 3 months, 4 subjects (1.6\%) had very poor appetite; 27 (11\%), moderately poor appetite; and $214(87.3 \%)$ had no significant appetite change. In the last 3 months, 124 subjects (50.6\%) showed no weight loss, 39 (15.9\%) lost 1-3 kg, 14 (5.7\%) lost more than $3 \mathrm{~kg}$, and 67 (27.8\%) were not aware of any changes. 7 subjects $(2.9 \%)$ were bed or wheelchair bound, $14(5.7 \%)$ were able to get out of the bed or wheelchair but lacked free mobility, and 224 (91.4\%) were mobile. In the last 3 months, 24 subjects (9.8\%) experienced psychological stress, and $221(90.2 \%)$ experienced no psychological stress. 7 subjects $(2.9 \%)$ had severe dementia or depression; 8 (3.3\%), mild dementia; and 230 (3.9\%), no mental problems. 35 subjects $(14.3 \%)$ had $\mathrm{BMI}<19,43$ (17.6\%) had $19 \leqq \mathrm{BMI}<21,69(28.2 \%)$ had $21 \leqq \mathrm{BMI}<23$, and 96 (40\%) had BMI $\geqq 23.217$ subjects $(88.6 \%)$ were able to live independently, and 28 (11.4\%) were dependent on others. 61 subjects (24.9\%) required more than 3 kinds of prescription drugs daily while $184(75.1 \%)$ did not. 25 subjects $(10.2 \%)$ had bedsores or skin ulcers while $220(89.8 \%)$ had intact skin. 78 subjects $(31.8 \%)$ were able to eat 3 complete meals a day; 128 (52.2\%), only two meals; and 39 (15.9\%), only one meal. In terms of protein intake, 117 subjects (47.8\%) were able to consume one serving of dairy per day (milk, cheese, yogurt) while $128(52.2 \%)$ could not. 205 subjects $(83.7 \%)$ were able to consume two or more servings of beans or eggs per week while 40 (16.3\%) could not. 182 subjects (74.3\%) consumed some meat, fish or poultry daily while $63(25.7 \%)$ could not. 172 subjects $(70.2 \%)$ were able to consume two or more types of fruits and vegetables per day while $73(29.8 \%)$ could not. 80 subjects $(32.7 \%)$ were able to consume $>5$ cups of liquid per day; 106 (43.3\%), 3-5 cups; and 59 (24.1\%), <3 cups. 213 subjects $(86.9 \%)$ had no self-feeding difficulty; $26(10.6 \%)$, some self-feeding difficulty; and $6(2.4 \%)$ were unable to eat without assistance. 143 subjects (58.4\%) felt that they had no nutritional problems, 13 (5.3\%) felt malnourished, and 89 (36.3\%) were uncertain about their personal nutritional status. 125 subjects (51\%) felt healthy and on par with those around their age, 43 (17.6\%) felt better than those around their age, 21 (8.6\%) felt worse than those around their age, and 56 (22.9\%) were unaware of their health condition. In terms of mid-arm circumference (MAC), the MAC of 17 subjects (6.9\%) were $<21 \mathrm{~cm}, 80(32.7 \%)$ were $21-21.9 \mathrm{~cm}$, and $148(60.4 \%)$ were $\geqq 22 \mathrm{~cm}$. In terms of calf circumference (CC), the CC for 98 subjects (40\%) were $<31 \mathrm{~cm}$, and $147(60 \%)$ were $\geqq 32 \mathrm{~cm}$.

\section{Depression status}

The mean total depression score and standard deviation were 1.56 ( \pm 0.65). 194 subjects $(79.2 \%)$ were satisfied with their lives while 51 subjects (20.8\%) felt otherwise. 113 subjects (46.1\%) had given up numerous activities and hobbies while 132 subjects (53.9\%) had not. 78 subjects $(31.8 \%)$ felt that their lives were empty while 167 subjects (68.2\%) felt otherwise.

95 subjects (38.8\%) often felt troubled while 150 subjects (61.2\%) felt otherwise. 161 subjects (65.7\%) often felt cheerful while 84 subjects (34.3\%) felt otherwise. 116 subjects $(47.3 \%)$ were fearful of misfortune while 129 subjects $(52.7 \%)$ had no such fear. 176 subjects $(71.8 \%)$ felt happy most of the time while 69 subjects $(28.2 \%)$ felt otherwise. 79 subjects (32.2\%) often felt helpless while 166 subjects (67.8\%) did not feel such helplessness. 84 subjects $(34.3 \%)$ preferred to stay home in the evening rather than go out to do something new while 161 subjects (65.7\%) felt otherwise. 101 subjects (41.2\%) felt that they had more memory problems than others while 144 subjects (58.8\%) felt otherwise. 217 subjects ( $88.6 \%$ ) felt that it is good to be alive while 28 subjects (11.4\%) felt otherwise. 47 subjects (19.2\%) felt useless while 198 subjects (80.8\%) felt otherwise. 130 subjects (53.1\%) felt energetic while 115 subjects (46.9\%) felt otherwise. 39 subjects (15.9\%) felt hopeless about their situation while 206 subjects (84.1\%) felt 
otherwise. 128 subjects (52.2\%) felt that others are better off than themselves while 117 subjects (47.8\%) felt otherwise.

\section{Cognitive functioning status}

The mean total cognitive functioning score and standard deviation were 9.13 ( \pm 1.37). 233 subjects (95.1\%) showed normal mental functioning; 6 subjects $(2.5 \%)$, mild cognitive impairment; 5 subjects (2.0\%), moderate cognitive impairment; and 1 subject $(0.4 \%)$, severe cognitive impairment.

\section{Cognitive correlation}

Gender (male and female) showed no significant correlation with total cognitive score $(\mathrm{t}=1.913)$. Education (divided into illiterate, elementary school, middle school, high school and university or above) showed no significant correlation with total cognitive score $(\mathrm{F}=0.449)$.
Self-reported health condition (divided into very unhealthy, unhealthy, average health, healthy and very healthy) showed no significant correlation with total cognitive score $(\mathrm{F}=0.228)$. Regular trans theoretical exercise (divided into more than 6 months, less than 6 months, agreed to exercise regularly, did not agree to exercise regularly) showed no significant correlation with total cognitive score $(\mathrm{F}=1.449)$. Smoking (divided into never smoked, smoked occasionally, smoked daily, had stopped smoking) showed no significant correlation with total cognitive score $(\mathrm{F}=3.034)$. The number of chronic diseases showed significant negative correlation $\left(r=-.204^{* *}\right)$, indicating that subjects with more chronic diseases had lower cognitive scores. Total cognitive score showed significant positive correlation with nutrition $\left(\mathrm{r}=.370^{\star *}\right)$, indicating that the elderly with better nutritional status had better cognitive functioning. Total cognitive score showed negative correlation with depression $\left(r=-.272^{\star *}\right)$, indicating that those with higher depression score had poorer cognitive functioning (Table 1).

\begin{tabular}{|l|l|l|l|l|l|l|}
\hline & Age & $\begin{array}{l}\text { Chronic diseases } \\
\text { number }\end{array}$ & Nutrition score & Depression score & Cognition score & Regular movement \\
\hline Age diseases & 1 & & & & \\
\hline $\begin{array}{l}\text { Chronic } \\
\text { number }\end{array}$ & $.165^{* *}$ & 1 & & & \\
\hline Nutrition score & $-.161^{*}$ & $-.342^{* *}$ & 1 & & & \\
\hline Depression score & 0.084 & $-.350^{* *}$ & $-.437^{* *}$ & 1 & & \\
\hline Cognition score & -0.95 & $-.204^{* *}$ & $.370^{* *}$ & $-.272^{* *}$ & 1 & \\
\hline Regular movement & -0.059 & -0.058 & $.131^{*}$ & $.139^{*}$ & 0.01 & 1 \\
\hline
\end{tabular}

Table 1: Pearson Correlation of Continuous Variables ( $\mathrm{N}=245)$.

\section{Conclusions and recommendations}

This study showed that most of the subjects, 233 (95.1\%), had normal mental functioning; 6 (2.5\%), had mild cognitive impairment; 5 (2.0\%), showed moderate cognitive impairment; and $1(0.4 \%)$, suffered severe cognitive impairment. Overall, important variables affecting cognition were the number of chronic diseases, total depression score and total nutrition score. As such, the study proposed many recommendations.

\section{Elderly with higher total nutrition score (and also higher cognitive functioning)}

Community health bureaus can organize more lectures on nutrition, provide knowledge-based health education and post relevant knowledge on official health center websites to encourage the elderly to spontaneously seek information and help them understand how to fulfill their nutritional needs so that they can improve their cognitive functioning.

\section{Elderly with higher total depression scores (lower cognitive functioning)}

Activity centers, out-reach bases and community colleges for the elderly can be established to provide active community environments to help elderly staying at home for long periods of time without any companions to get out of the house. In addition, a depression scale should be administered regularly to strengthen their cognitive functioning.

\section{Elderly with more chronic diseases (lower cognitive functioning)}

Encourage the elderly to receive regular assessment and screening, provide individual care, track records, encourage regular exercise and evaluate for obesity causing environments in order to reduce the number of chronic diseases in the elderly and increase their cognitive functioning.

\section{Research Limitations}

1. Due to limited human resource, time and funding, as well as the need to fulfill the survey objective and special needs, convenience sampling was adopted. Therefore the findings are applicable only to the study population.

2. This study adopted a questionnaire survey that required subject recall. Therefore, the survey findings may be inaccurate due to recall bias.

\section{Suggestions for Future Research}

The study population was limited to the elderly living in northern Taiwan, and hence results cannot be generalized to the entire elderly population in Taiwan. Other influential variables have yet to be 
Citation: Chang CF, Kuo TH (2018) Impact of Nutrition and Depression on the Cognitive Functioning of the Elderly in Taiwan. J Aging Sci 6: 197. doi:10.4172/2329-8847.1000197

Page 5 of 5

delineated, and hence future research samples can be extended to different regions. By adopting a longitudinal or qualitative study, the inferences presented by the study results can be further analyzed to provide early prevention and improve cognitive dysfunction.

\section{References}

1. Jong-Ling Fuh (2008) Dementia in Taiwan: Current Status. Taiwan Geriatr Gerontol 3: 169-181.

2. Chia-Jung Hsieh (2003) Application and measurement of cognitive function in the elderly. J Health Science 5: 387-395.

3. Ka-Ian Leong, Yen-Ching Chen, Jen-Hau Chen (2014) Dementia: A focused review. J Int Med Taiwan 25: 151-157.

4. Eaton WW, Anthony JC, Gallo J, Cai G, Tien A, et al. (1997) Natural history of Diagnostic Interview Sched/DSM-IV major depression: The Baltimore Epidemiologic Catchment Area follow-up. Arch Gen Psychiatry 54: 993-999.
5. Alzheimer's Disease International (2015) World Alzheimer Report 2015: The Global Impact of Dementia: The incidence of dementia. pp: 33

6. Taiwan Alzheimer's Disease Association (2017) What does the mentally retarded population know?).

7. Report on 2nd ICAH-NCGG Symposium (2016).

8. World Health Organization (2012). Active ageing: a policy framework. Geneva: World Health Organization.

9. Tzu-Chuan C, Hsiu-Chi H, Zong-Sing L (2013) Effects of 12-wk Sitting Tai Chi Chuan training on lower-extremity muscle strength and activities of daily living performance in elderly patients with dementia. Taiwan J Gerontol Health Res 9: 20-35

10. Yeh TL, Liao IC, Yang YK, Ko HC, Chang CJ, et al. ( 1995) Geriatric depression scale (Taiwanese and Mandarin translations) Clinic Gerontol 15: $58-60$

11. Chun-Hsiang Yu (2009) Population-specific anthropometric cut-points improve the predictive ability of the Short Form Mini Nutritional Assessment (MNA-SF). 